\title{
A Novel Dynamic Splitting Method for Production Based on Material Balance Theory and Catastrophe Theory in Tight Gas Reservoirs
}

\author{
Shengye Hao, Xinyu Qiu, Pengcheng Liu $\mathbb{D}^{\text {, }}$, and Xiaoxia Chen \\ School of Energy Resources, China University of Geosciences (Beijing), Beijing 100083, China \\ Correspondence should be addressed to Pengcheng Liu; liupengcheng8883@sohu.com
}

Received 31 March 2021; Accepted 11 June 2021; Published 24 June 2021

Academic Editor: Jianhui Yang

Copyright (C) 2021 Shengye Hao et al. This is an open access article distributed under the Creative Commons Attribution License, which permits unrestricted use, distribution, and reproduction in any medium, provided the original work is properly cited.

\begin{abstract}
Splitting methods play a significant role in the coproduction of tight reservoirs which are characterized by vertical multilayer superimposition. It directly affects the accuracy of reservoir performance analysis and detailed descriptions. However, conventional splitting methods are limited to a few factors and static factors without considering the effect of layer parameter change. In this study, sensitivity analysis was carried out on five factors that affect the production splitting in coproduction wells. The research shows that in the production process, multiple parameters have a direct impact on the production of layers. Different parameters, which have to be included to split production, have different scale effects on layer production. Comparing the results of the $\mathrm{KH}$ method with the numerical simulation results, the limitation of the $\mathrm{KH}$ method for yield splitting is illustrated. A novel dynamic splitting method for production (DPSM) was proposed. This method is based on two primary methods, which are the multifactor static method for production splitting of gas (GPSM) and water (WPSM) and use the catastrophe theory and material balance equation (MBE) and obtain the final results by iterative method. The advantage of this method is that more accurate results in the production process are obtained by selecting eight factors, which contain 6 static factors and 2 dynamic factors, for research. It is more in line with the production practice that the ultimate results of production splitting vary with the production process. The accuracy and practicality of the results had been verified by numerical simulation. This method has practical significance for production splitting in tight gas reservoirs.
\end{abstract}

\section{Introduction}

Tight gas reservoirs are the main targets of unconventional development [1-3]. China has shown great enthusiasm for developing tight gas reservoirs $[4,5]$. The tight gas reservoirs, which are characterized by vertical multilayer superimposition, are often developed by the multilayer coproduction technology $[6,7]$. However, multilayer coproduction will lead to difficulty of reservoir dynamic analysis and detailed descriptions and will affect the formulation of production measures [8-10]. Thus, an accurate production splitting method is needed for the commingled production well.

The conventional method of production splitting is to obtain geological parameters such as permeability and effective thickness for qualitative research without suspending production. At present, conventional production splitting techniques focus on four types of methods: parameter methods, numerical simulation methods, production profile testing methods, and other improved methods derived from these methods.

The traditional parameter method named $\mathrm{KH}$ is mainly to split production by permeability $(\mathrm{K})$ and effective thickness $(\mathrm{H})$. Only two static factors are considered in this method. There is a big deviation in the calculation results. Many scholars have modified this method: the KNK method considers well-controlled geological reserves $(\mathrm{N})$ in layers [11], the $\mathrm{KHK}_{\mathrm{r}}$ method considering effective permeability $\left(\mathrm{K}_{\mathrm{r}}\right)$ [12-15], and the production splitting method combining the $\mathrm{KH}$ method with the production profile testing method [16]. These methods improve the accuracy of the 
method at different levels, but it still cannot meet the practical application of gas reservoirs due to their only consideration of single factors and static factors.

Numerical simulation is an important means to understand the reservoir. Through history simulation, the properties and states can be obtained, and then the production of the reservoir can be split in each stage of development. With the development of computer technology, numerical simulation has developed greatly. Prabowo et al. were the first to study the dynamic response of well production and pressure by establishing a numerical simulation model without layer interflow [17]. Later, other scholars studied the effects of starting pressure gradient on the production of layers through numerical simulation [18]. The application of numerical simulation in splitting has great vitality. However, the results cannot be directly applied to production due to the multisolution.

The production profile test method uses combining logging tools to record the practical production rates of each section. Then, we can obtain the splitting coefficient of reservoir production by interpreting the recorded data. Generally, this method is the most accurate [19]. But the testing process will affect normal production, and the disadvantage of time consuming and high cost also leads to hesitation about this method. For these reasons, the method of profile testing has not been applied widely in practical production.

Researchers have proposed many new production splitting methods on the basis of various mathematical theories and basic seepage laws [20-24]. Hu et al. used grey relational analysis to analyze the various weights that affect the split of production [25]. Rasoul et al. came up with a method to calculate the splitting coefficient by temperature [26]. Gu et al. established a static method by utilizing the catastrophe theory $[27,28]$. This method took into account a number of factors that affect the splitting coefficient. In the initial production stage, the calculation results showed certain accuracy. However, the data adopted in this method were all static data at a certain moment, which cannot be applied to the entire production process. Mi et al. improved on this method according to the fractional flow equation to establish a dynamic splitting method [29]. This method showed the feasibility of incorporating dynamic data into the splitting method based on the catastrophe theory. But the calculation results of the fractional flow equation were limited to the parameters around the well and cannot accurately describe the dynamic changes of the entire reservoir. So, other ideas are needed to incorporate dynamic data into the splitting method.

The above method that we discussed cannot well combine dynamic and static data in the calculation process. In addition, most of the methods also do not consider the effect of water production. The calculation results are either complicated or cannot be consistent with the practical results of dynamic changes. Therefore, a simple and accurate method is urgently needed in the practical production splitting process.

In this paper, the results of sensitivity analysis show that the production splitting is controlled by multiple factors and it changes dynamically over time. A novel and more accurate dynamic splitting method considering dynamic and static factors was proposed based on catastrophe theory, MBE, and iterative calculation. It is proved that DPSM is more accurate and more practical for tight gas reservoir by comparing with the $\mathrm{KH}$ method and original splitting method based on the catastrophe theory.

\section{Theoretical Method}

In the process of production, the production of layers is jointly affected by many factors. The variation of subsurface parameters will also lead to the error of splitting [30-34]. To avoid this error, DPSM was proposed. Cumulative water production of each layer at the first time step can be obtained by both WPSM and the production of commingled wells. Then, the instantaneous saturation of gas and water can be obtained by MBE at the second time step. We can get the instantaneous permeability of gas and water by the relative curve. The instantaneous splitting coefficient of gas and water, at the second time step, is obtained, by applying the instantaneous data into GPSM and WPSM, respectively. Moreover, we can split the production at any time by iterating. A conceptual model of a commingled producing well based on real tight gas reservoir is established to effectively verify the accuracy of this method.

In this method, the catastrophe theory, material balance equation, and iterative calculation method are used in the calculation process. Calculation takes into account 2 dynamic factors: gas saturation and effective permeability and 6 static factors: effective thickness, porosity, perforating height, sandstone content, reservoir density, and gas reservoir depth. The rationality and accuracy of this method are guaranteed by the multifactor analysis method and the comprehensive application of various theories. It needs to be highlighted that the iterative calculation produces splitting results at each instantaneous moment, and the results are more in line with the reality of dynamic changes of parameters in practical production.

There are some assumptions that need to be specified in the calculation. It is considered that the layer production is controlled by the same factors during the calculation. The effect of different fluid properties on production is ignored. Most importantly, we do not consider communication between layers, so the influence factors act independently in the layers.

\subsection{Production Splitting Method Based on the Catastrophe Theory}

2.1.1. Catastrophe Theory. The catastrophe theory assumes that the states of catastrophe events are determined by a number of state variables [35]. A state variable is controlled by one or more control variables. A number of related control variables and state variables can constitute a catastrophe system. If the external conditions change in a system, we can describe the relationship between control variables and state variables. There are some potential functions $(f(x))$ that can express the interaction between disparate variables and disparate states of the system [36]. The critical points are going to be gotten by the derivative of $f(x)$. All critical points in the system can form an equilibrium surface. The events, which are not on the equilibrium surface of the system, are going to be the sudden change catastrophe event. It is conventional 
to use the bifurcation to reflect the relationship between state variables and each control variable. The bifurcation refers to small changes in system parameters, resulting in sudden changes. We can express the bifurcation equation by state variables which reflect the relationship between state variables and control variables. The time is ripe for deriving the normalization formula to analyze the catastrophe system, which aims to domesticate the different mass states of the nodes to the same comparable mass state. The normalization formula and bifurcation equation are fixed mathematical expressions.

The most commonly used models of the catastrophe theory include fold catastrophe, cusp catastrophe, swallowtail catastrophe, oval umbilicus catastrophe, hyperbolic catastrophe, butterfly catastrophe, and parabolic catastrophe. In this paper, we just use cusp catastrophe (equations (1) and (2)) and swallowtail catastrophe (equations (3) and (4)). Equation (1) represents the interaction between systems when there are two control variables and one state variable of the system, and equation (2) is a normalization formula for the comprehensive analysis and judgment of whether the system has changed suddenly. Similarly, equations (3) and (4) express the same meanings while the number of control variables has changed to two.

$$
\begin{aligned}
x_{u} & =\sqrt{u}, \\
x_{v} & =\sqrt[3]{v}, \\
u & =-6 x^{2}, \\
v & =-8 x^{3}, \\
x_{u} & =\sqrt{u} \\
x_{v} & =\sqrt[3]{v} \\
x_{w} & =\sqrt[4]{w}, \\
u & =-6 x^{2}, \\
v & =8 x^{3}, \\
w & =-3 x^{4} .
\end{aligned}
$$

2.1.2. Production Splitting Method of Gas (GPSM) and Water (WPSM). In this study, we decompose the overall target to construct a multilevel architecture diagram by studying the state variables and control variables of the event. Normalization formula of different models are used to quantify the operation. The final evaluation result of the multilevel architecture diagram is obtained.

The layer production of multilayer commingled producing wells is regarded as controlled by three control variables, which are reserve characteristics, development characteristics, and geological characteristics. These control variables are controlled by several factors as state variables at the same time, which constitute a subsystem. The reserve characteristics are controlled by effective thickness, , porosity and gas saturation, which constitute the swallowtail catastrophe model. The development characteristics are controlled by two control variables, effective permeability, and perforating height, which constitute the cusp catastrophe model. The geological characteristics are controlled by the sandstone content, reservoir density, and gas reservoir depth, which constitute the swallowtail catastrophe model. Reservoir characteristics, development characteristics, and geological characteristics constitute the swallowtail catastrophe model.

In this study, it is assumed that the influencing factors of gas production and water production of layers are the same. Moreover, we can use the same factors to build similar systems of catastrophe analysis for gas and water. Figure 1 displays the schematic of the catastrophe system for gas and water. The markings in Figures 1(a)-1(c) represent the parameters at the positions in the catastrophe system and do not represent determined parameters.

The equilibrium surface is constituted by choosing the most disadvantage of factors. Maximum values of some affecting factors which have a positive effect on layer production are selected, e.g., the thickness, porosity, saturation, and effective permeability in the reservoir. The other factors are negative to the production and are selected as the minimum, for example, perforating height, sandstone content, reservoir density, and gas reservoir depth. So, we can get the equilibrium surface successfully.

The effect factors of the system need to be normalized so as to simplify the complexity of subsequent calculation (equation (5)). $x$ is the normalized result value, $x_{i}$ is the influence factor value of each system, and $c_{i}$ is the maximum of the influence factor of each system.

$$
x=\frac{x_{i}}{c_{i}} .
$$

The weight of each factor in the subsystem of reserve characteristics, development characteristics, and geological characteristics can be calculated by the normalization formula. The weighted average value for factor weight in the subsystem is calculated, so that we can get the object value to the whole system. Similarly, the final object value of the system is obtained by the normalization formula and weighted average method, which is the weight of the comprehensive factor in each layer. The calculation formula is shown below (equations (6)-(13)).

$$
\begin{gathered}
x_{A_{1}}=\left(A_{1}\right)^{1 / 2}, \\
x_{A_{2}}=\left(A_{2}\right)^{1 / 3}, \\
x_{A_{3}}=\left(A_{3}\right)^{1 / 4}, \\
x_{A}=\frac{\left(x_{A_{1}}+x_{A_{2}}+x_{A_{3}}\right)}{3}, \\
x_{B_{1}}=\left(B_{1}\right)^{1 / 2}, \\
x_{B_{2}}=\left(B_{2}\right)^{1 / 3}, \\
x_{B}=\frac{\left(x_{B_{1}}+x_{B_{2}}\right)}{2},
\end{gathered}
$$




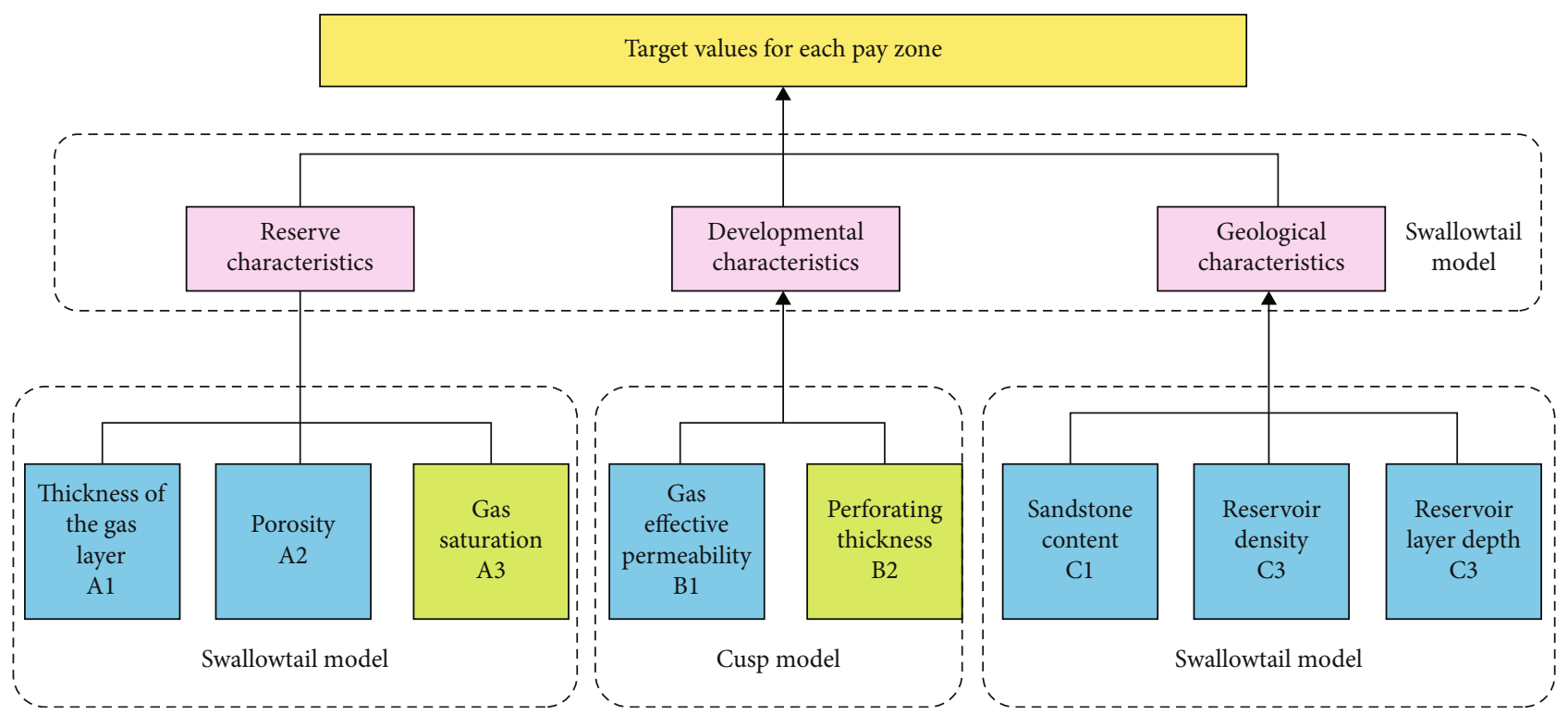

(a) The catastrophe system of GPSM

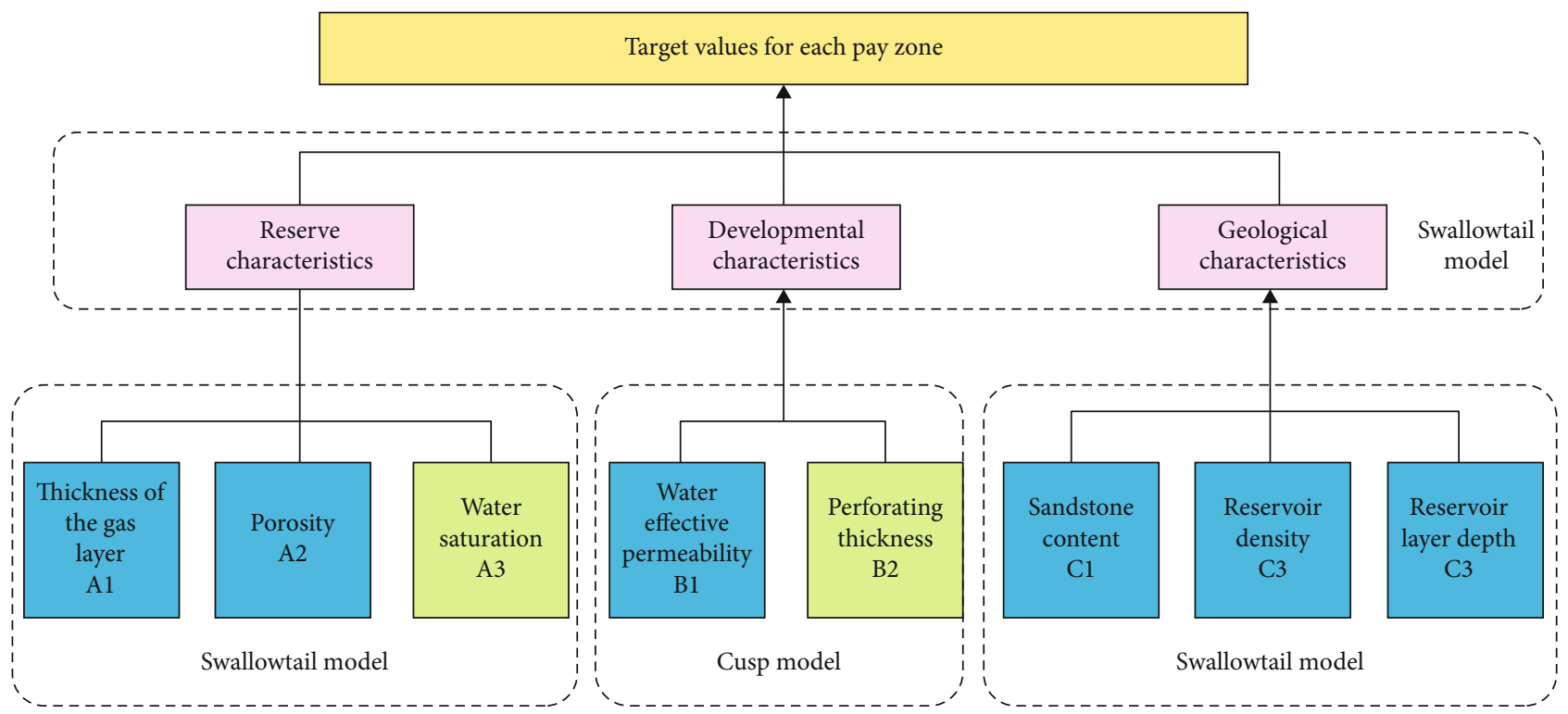

(b) The catastrophe system of WPSM

FIGURE 1: The schematic of the catastrophe system for gas and water. Final target values are yellow, development feature target values are pink, static variables are blue, and dynamic variables are green.

$$
\begin{gathered}
x_{C_{1}}=\left(C_{1}\right)^{1 / 2}, \\
x_{C_{2}}=\left(C_{2}\right)^{1 / 3}, \\
x_{C_{3}}=\left(C_{3}\right)^{1 / 4}, \\
\mathrm{x}_{\mathrm{C}}=\frac{\left(\mathrm{x}_{\mathrm{C}_{1}}+\mathrm{x}_{\mathrm{C}_{2}}+\mathrm{x}_{\mathrm{C}_{3}}\right)}{3}, \\
x_{F_{A}}=\left(F_{A}\right)^{1 / 2}, \\
x_{C_{2}} x_{F_{B}}=\left(F_{B}\right)^{1 / 3}, \\
x_{C_{2}} x_{F_{C}}=\left(F_{C}\right)^{1 / 4},
\end{gathered}
$$

$$
x_{F}=\frac{\left(x_{F_{A}}+x_{F_{B}}+x_{F_{C}}\right)}{3} .
$$

The production weight of layers by comparing the final object value of layers and the equilibrium surface can be obtained. The production weight of layers is the splitting coefficient which was calculated by the catastrophe theory. The splitting coefficient calculation is shown in equation (14) as follows:

$$
S_{i}=\frac{x_{F_{i}}-x_{F}{ }^{\prime}}{\sum_{i=1}^{n}\left(x_{F_{i}}-x_{F}^{\prime}\right)}
$$




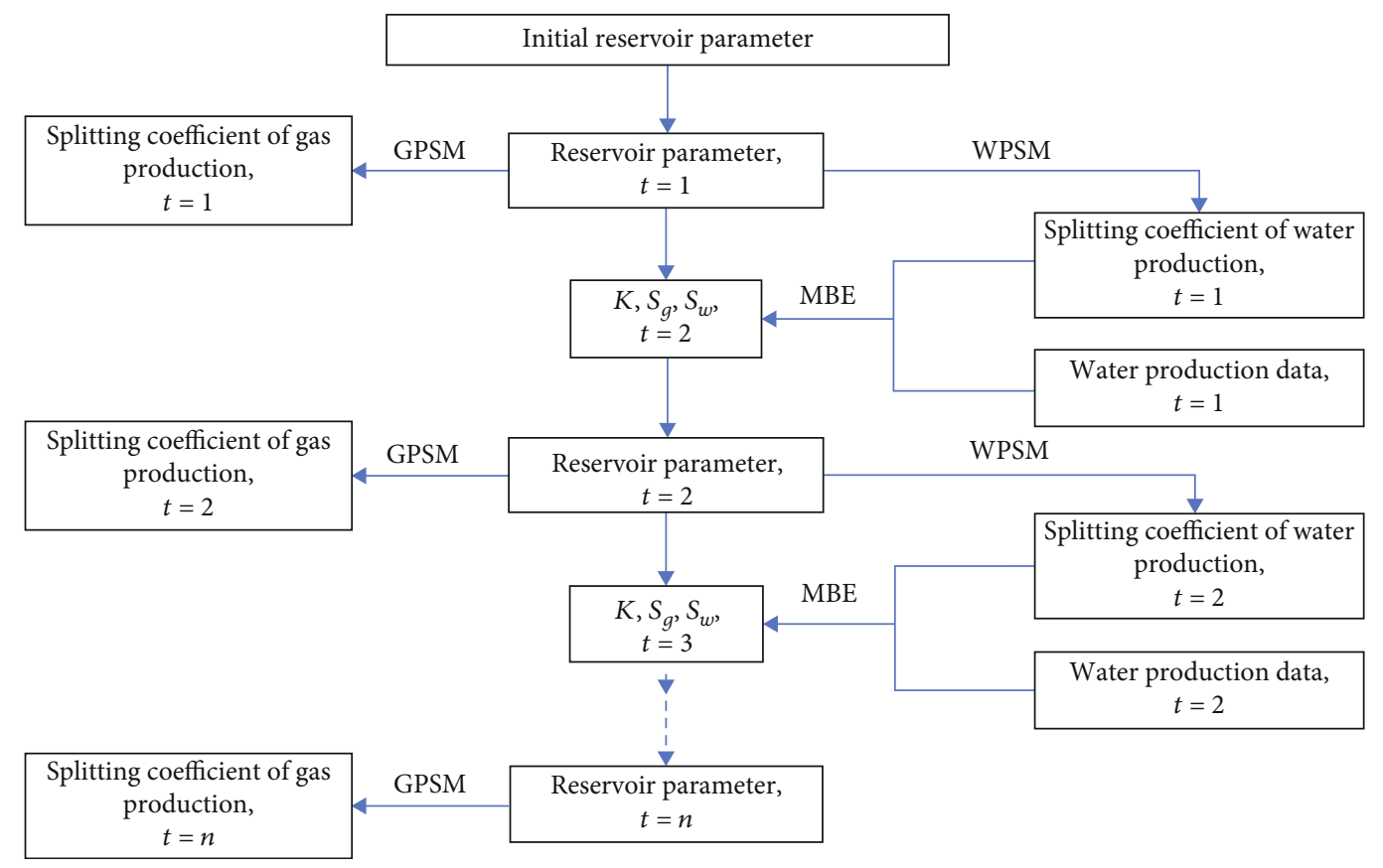

FIGURE 2: Calculation process of DPSM.

The WPSM and GPSM have the same calculation process due to the similar catastrophe system. We can use the same formula when using WPSM.

2.2. Calculation of Dynamic Data. The material balance theory is one of the basic theories to study reservoir flow and many scholars have optimized the MBE in many ways [37]. It is not difficult to calculate the dynamic data by MBE if the initial layer reserves are known. The initial data of the reservoir is measured by the well log and well test of the layer. Based on these data, the average water saturation of the layer can be obtained easily at each moment by $\mathrm{MBE}$. The required material balance equation is shown in equation (15) as follows:

$$
\begin{gathered}
S_{g}=S_{g i}+B_{w i} \frac{G_{w}}{A h \phi}, \\
K_{g}=K_{r g} \times K .
\end{gathered}
$$

The relative permeability curves of each core are obtained from experimental data and field well test interpretation results [38]. If we calculate the average saturation of the layer at each moment, the real-time relative permeability can be obtained through the relative permeability curve.

2.3. Dynamic Production Splitting Method (DPSM). Changes in parameters occur throughout the production process. The traditional splitting method of the catastrophe theory only works at the time nodes which are defined by the data point. It will show strong errors at other points in time. In this study, we mainly consider the variation of water saturation and effective permeability with the production process in tight gas production. Figure 2 demonstrates the calculation process.
In Figure 2, initial data of layers are firstly obtained from the well test and log and the splitting coefficient of water and gas can be calculated by WPSM and GPSM at the first time step, respectively. The time is ripe for calculating the water production of the layer. Instantaneous saturation of water and instantaneous relative permeability in the second time step can be obtained. The instantaneous splitting coefficient in the second time step can be calculated similarly by GPSM. Ultimately, we can obtain the splitting coefficient at any time by iterating.

2.4. Numerical Model for Coproduction. In order to obtain an accurate response of DPSM through simple calculation and simplify the complexity of numerical simulation, a homogeneous conceptual model is established which is characterized by a double layer. Figure 3 shows the morphology of the conceptual model. In Figure 3, a vertical well is in the center of the model. The design concept model is a $50 \times 50 \times 12$ grid distribution, in which the vertical $1 \sim 5$ layers of grids constitute layer 1 , the $6 \sim 7$ layers constitute impermeable interlayers, and the $8 \sim 12$ layers belong to layer 2 . The horizontal step size of the grid is set as $10 \mathrm{~m}$ and the longitudinal step size is set as $2 \mathrm{~m}$.

The effect of reservoir fluid on the production splitting is not discussed in this study. We assume that the fluids of layers have the same properties in this conceptual model. Fluid properties are set out in Table 1. The main points of the conceptual model are the difference between layers. We set the layers with different properties, such as initial water saturation, porosity, effective permeability, and medium depth of the gas layer. Table 2 lists the initial parameters of the two reservoirs. Figure 4 displays the gas-water relative permeability curve of this model.

Figure 5 displays the effect of the wellhead production rate on splitting. In Figure 5, with the increase of the gas 


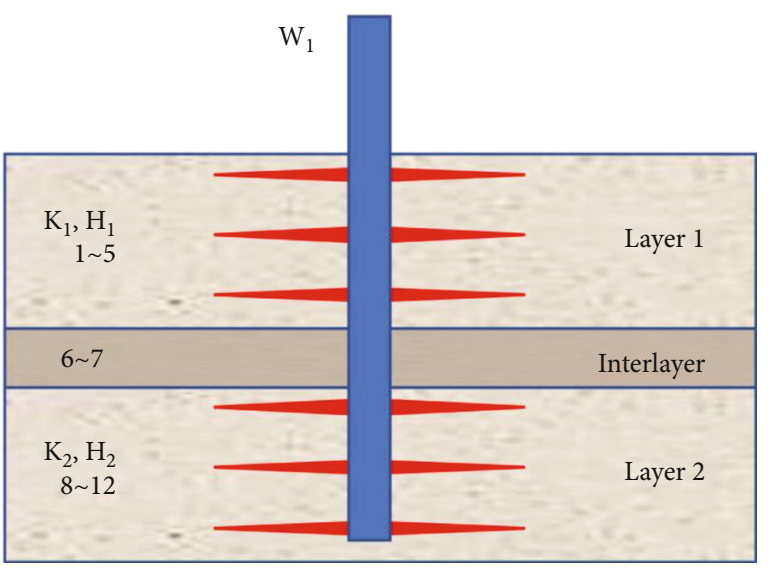

FIgURE 3: Concise diagram of the mechanism model.

TABLE 1: Key parameters of the fluid properties.

\begin{tabular}{lc}
\hline Parameter & Value \\
\hline Water compression coefficient $\left(\mathrm{MPa}^{-1}\right)$ & $3.92 \times 10^{-4}$ \\
Water viscosity $(\mathrm{cP})$ & 0.476 \\
Water density $\left(\mathrm{kg} / \mathrm{m}^{3}\right)$ & 1000 \\
Gas density $\left(\mathrm{kg} / \mathrm{m}^{3}\right)$ & 0.761 \\
\hline
\end{tabular}

TABLE 2: The initial parameters of the two reservoirs.

\begin{tabular}{lcc}
\hline Layer & Layer 1 & Layer 2 \\
\hline The thickness of the gas layer $(\mathrm{m})$ & 10 & 10 \\
Gas saturation $(\%)$ & 60 & 55 \\
Porosity $(\%)$ & 12 & 8 \\
Perforating height $(\mathrm{m})$ & 10 & 10 \\
Effective permeability $(\mathrm{mD})$ & Dynamic calculation \\
Sandstone content $(\%)$ & \multicolumn{2}{c}{ result } \\
Reservoir density $\left(\mathrm{g} / \mathrm{cm}^{3}\right)$ & 2.36 & 81 \\
Middle depth of layer $(\mathrm{m})$ & 2305 & 2.36 \\
Absolute permeability $(\mathrm{mD})$ & 0.08 & 2319 \\
\hline
\end{tabular}

production rate, the effect of the rate will gradually disappear. In this paper, in order to eliminate the effect of the gas production rate on the splitting coefficient of layer production, the gas production rate is set as a value as large as possible which conforms to reality. The gas production rate is set at $5000 \mathrm{~m}^{3} /$ day.

\section{Results and Discussion}

3.1. Sensitivity Analysis of the Multiple Factor. Factors used in DPSM are analyzed by numerical simulation to illustrate the necessity of considering the process of production splitting. The layer production ratio is affected by the fluid flow process, which proves the necessity of studying the dynamic parameters. This study also proves the limitations of the

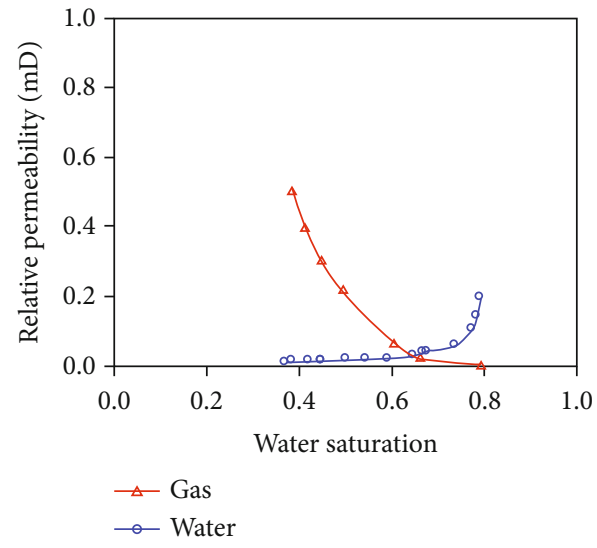

FIGURE 4: Gas-water relative permeability curve.

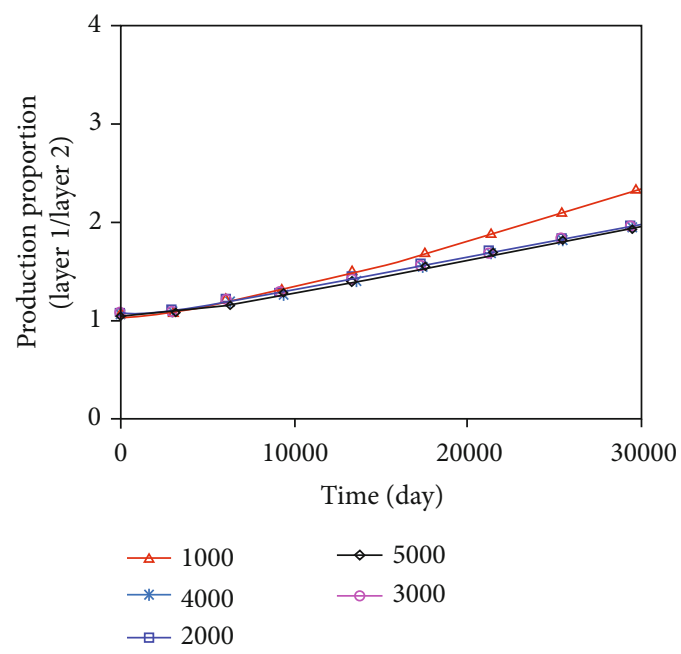

FIgURE 5: The effect of the wellhead production rate on splitting.

$\mathrm{KH}$ method and indicates that a dynamic production splitting method with multifactor analysis is needed.

3.1.1. Permeability. The effect of permeability of layers on the production ratio of the coproduction well is analyzed by the single-factor control method. We set the upper permeability as a constant value of $0.12 \mathrm{mD}$ and set the lower layers as $0.30 \mathrm{mD}, 0.40 \mathrm{mD}$, and $0.60 \mathrm{mD}$ to achieve the purpose of setting the permeability ratio of two layers as 2,3 , and 4 , respectively. Figure 6(a) displays the effects of permeability.

In Figure 6(a), the results show that the production ratio is close to the ratio of layer permeability. The curve of the production ratio tends to be the ratio of permeability with the development of layers. When the ratio of permeability is small, there is no obvious change in the production process. When the ratio of permeability is larger, the variation range is larger.

Permeability determines the flow capacity of fluid. Through the analysis of permeability, it is found that the permeability of layers is an important factor affecting the ratio of layer production. With the increase of permeability difference, the ratio of production is larger. We also found that 

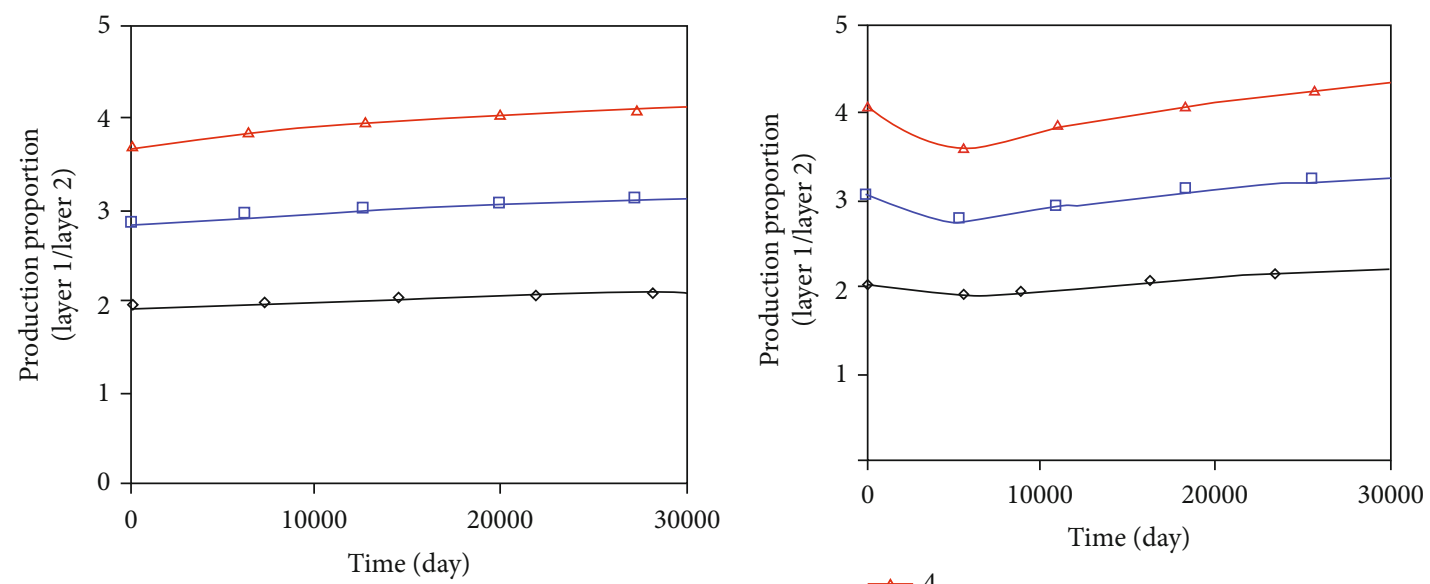

$$
\begin{aligned}
& \triangle 4 \\
& \square-3 \\
& \multimap 2
\end{aligned}
$$

$$
\begin{aligned}
& \triangle 4 \\
& \square-3 \\
& \multimap 2
\end{aligned}
$$

(a)

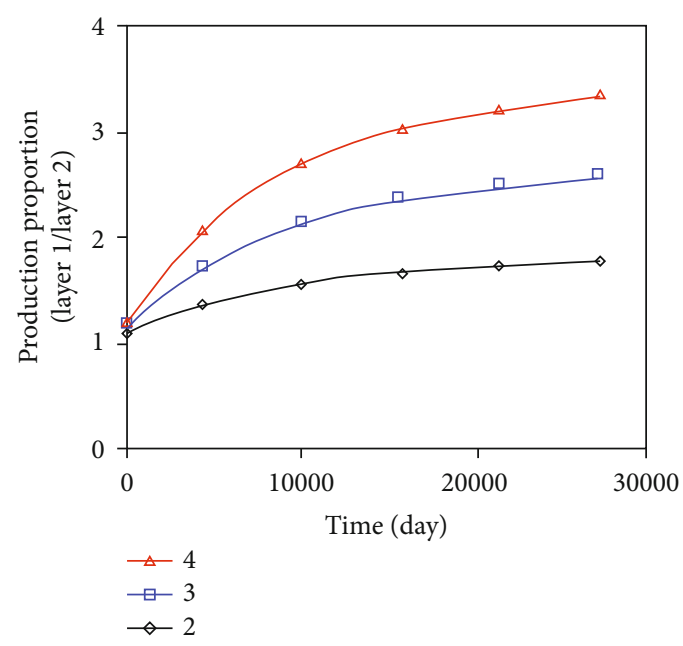

(c) (b)

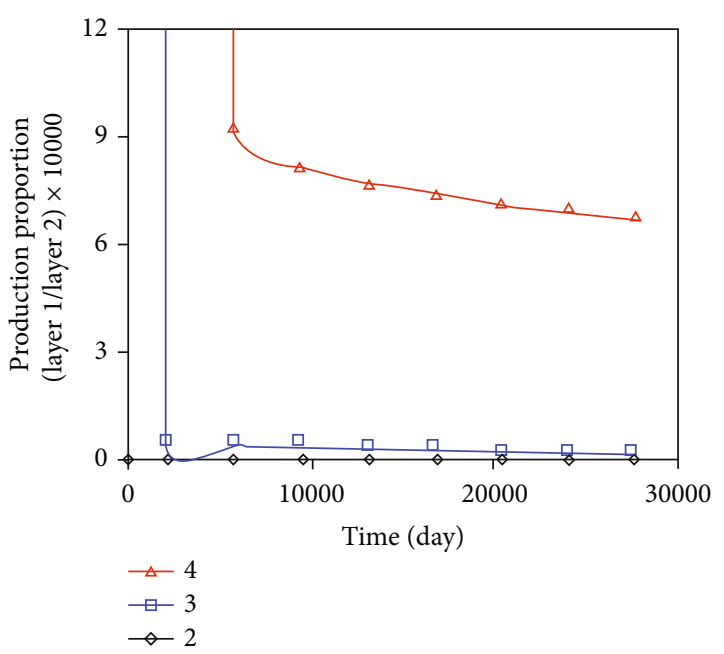

(d)

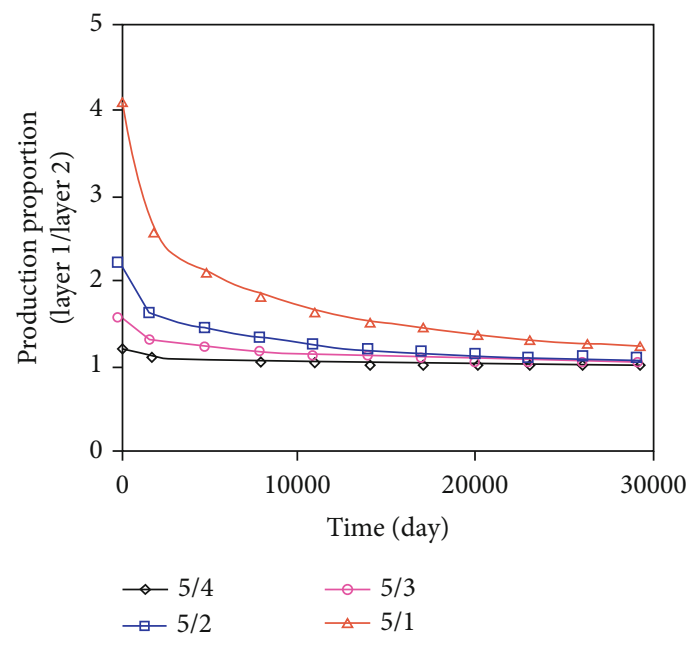

(e)

Figure 6: Sensitivity analysis. (a) The effects of permeability, (b) the effect of effective thickness, (c) the effect of porosity, (d) the effect of saturation, and (e) the effect of perforation thickness. 
when the permeability ratio of the upper and lower layers is constant, the production ratio of the layer still changes. So, we think that there are other factors that affect the production ratio of layers. It is necessary to study other factors which affect the production ratio. It has great significance for the production splitting.

3.1.2. Effective Thickness. We set the same parameters in the layers of the conceptual model and only change the value of effective thickness to achieve the purpose of studying the sensitivity of effective thickness. We set the upper effective thickness as a fixed value of $2 \mathrm{~m}$ and set the lower layer as $0.66 \mathrm{~m}$, $0.50 \mathrm{~m}$, and $0.40 \mathrm{~m}$ to achieve the purpose of setting the effective thickness ratios of two layers as 2,3 , and 4 . As shown in Figure 6(b), with the passage of time, the production ratio of the layers has a fluctuation change which first decreases and then increases. The higher the effective thickness ratio is, the higher the production ratio increases in the later period.

The effective thickness represents the size of the production section and the size of the reserves of layers. This directly controls the production ratio near to the ratio of effective thickness. In the production process, the gas near the well is produced before the pressure reaches the boundary. The dominant layer has a large rate of production and needs more replenishment. However, the gas flow in layers is slow and cannot be replenished quickly and the production ratio drops at the beginning. After a period of time, the production ratio increases due to the increased flow provided by the layers. This sensitivity analysis indicates that the effective thickness is also an important factor controlling the layer production ratio. The fluid flow stage also affects the production ratio of the layers. We must consider the effect of the fluid flow and effective thickness when splitting the production.

3.1.3. Porosity. Through the method of controlling variates, only the porosity of layers in the conceptual model is set to change and the effect of the porosity change on the production splitting is observed. We set the upper porosity as a fixed value of 0.12 and set the lower layers as $0.60,0.40$, and 0.30 to achieve the purpose of setting the porosity ratios of two layers as 2, 3, and 4, respectively. As shown in Figure 6(c), the production ratio is close at the early production stage. With the development of the reservoir, the ratio of production rises and tends to be a stable value. Under the influence of different porosity, the difference of ratio tends to be stable.

These changes are due to the reserves controlled by porosity. After reserves near the wellbore are exhausted, reserves far away from the wellbore have started to be tapped. High-porosity reservoirs provide faster. When the pressure wave reaches the boundary, the production of layers is directly controlled by reserves and the production ratio tends to be the ratio of reserves. This proves that the porosity of the reservoir can have a great effect on the production of layers. The effect of porosity must be considered when splitting production.

3.1.4. Saturation. Gas saturation in tight gas reservoirs is generally less than $60 \%$. We set the upper gas saturation as a fixed value of 0.60 and set the lower layers as $0.30,0.20$, and 0.15 to achieve the purpose of setting the gas saturation ratios of two layers as 2, 3, and 4, respectively. The effect of saturation on production splitting is analyzed by observing the influence of the initial gas saturation on the production ratio. As shown in Figure 6(d), at the beginning, the gas production ratio, in which the saturation ratios are 4 and 3 , tends to be infinite because layer 2 does not produce gas. After a period of time, layer 2 begins to produce gas and the relative ratio appears. In the continuation of production, the production ratio of the three curves has a downward trend.

In the early stage of production, due to the high water saturation, the effective permeability of the gas phase is too low to not flow out. After a period of time, layer 2 begins to produce gas due to the water saturation decreases. Because of the continuous decreases of water saturation, the relative permeability of gas in layer 2 gradually increases. The gas production increases, and the production ratio decreases. The large difference between the production ratio indicates that saturation has a great impact on layer production. We can indicate that the saturation is dynamic varying with the development, so it is necessary to consider its effects.

In the early stage of production, due to the high water saturation, the relative permeability of the gas phase is too low to not flow out. After a period of time, layer 2 begins to produce gas due to the water saturation decreases. Because of the continuous decreases of water saturation, the relative permeability of the gas phase in layer 2 gradually increases. The gas production increases, and the production ratio decreases. The large difference between the production ratio indicates that saturation has a great impact on layer production. We can indicate that the saturation is dynamic varying with the development, so it is necessary to consider its effects when splitting production.

3.1.5. Perforation Thickness. The effect of perforation thickness on practical production splitting was studied by setting the difference of perforation thickness. Moreover, in order to ignore the impact of different perforation positions on production, we chose the middle position as far as possible. The upper perforation thickness is set as a fixed value of 10 $\mathrm{m}$, and the lower layers were set as $8 \mathrm{~m}, 6 \mathrm{~m}, 4 \mathrm{~m}$, and $2 \mathrm{~m}$ to achieve the purpose of setting the gas saturation ratios of two layers as $5 / 4,5 / 3,5 / 2$, and 5/1, respectively. Figure 6(e) displays the result of numerical simulation.

In Figure 6(e), the layer production ratio appears to be significantly different at the initial stage. The value of the production ratio, which has a small ratio of perforation thickness, is close to 1 . When the perforation thickness ratio increases, it shows a huge difference in the yield ratio. In the later stage, the value of the production ratio all tends to be 1 . Due to the different perforation thickness, the size of the flow section is different. It leads to the difference of production in the early stage. In the later stages of production, as the remaining oil gradually decreases, the effect of perforation thickness on the production gradually decreases. The final phenomenon indicates that the value of the production ratio tends to be 1 . We can conclude that perforation thickness has an important effect on the production ratio and it must be taken into account in the production splitting. 


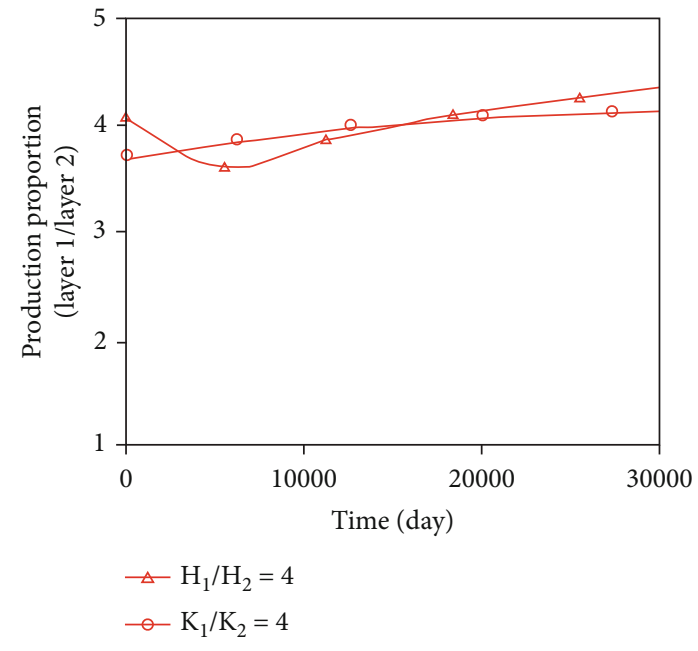

FIGURE 7: The numerical simulation results of controlling the same $\mathrm{KH}$ ratio, $\mathrm{K}_{1} \mathrm{H}_{1} / \mathrm{K}_{2} \mathrm{H}_{2}=\mathrm{H}_{1} / \mathrm{H}_{2}=\mathrm{K}_{1} / \mathrm{K}_{2}=4$.

The above research shows that in the production process, multiple parameters have a direct impact on the production of layers. Different parameters have different scale effects on layer production. In the process of production splitting, multiple factors are needed to be analyzed synthetically. The production splitting method constructed by considering many factors is more reasonable and accurate.

3.2. KH Method Validation. The KH method (equation (16)), which only considers permeability and effective reservoir thickness, does not consider the influence of multiple factors on the reservoir.

$$
p=\frac{K H}{\sum K_{i} H_{i}} .
$$

Numerical simulation results show that the ratio of production is not always the same as the ratio of $\mathrm{KH}$ in numerical simulation (Figures 6(a) and 6(b)). It changes dynamically with the production process. When the ratio of $\mathrm{KH}$ is the same, the numerical simulation results of the layer production ratio are also different.

Figure 7 displays the numerical simulation results of controlling the same KH ratio. These results show that the solution of numerical simulations is not the same as the ratio of $\mathrm{KH}$ and always fluctuates around the results of $\mathrm{KH}$. Even if the $\mathrm{KH}$ ratio is set to be the same, the numerical simulation results also show differences. This method only calculates the splitting coefficient based on permeability and effective thickness, which generates a rough result. There must be other factors controlling the production of layers in the combined well. Moreover, because the selected parameters are static parameters, they cannot be adapted to the change of the practical yield splitting coefficient in the production process. The error increases as the production process progresses. Therefore, dynamic analysis of the multiple factor is necessary to get more accurate results during the production splitting.

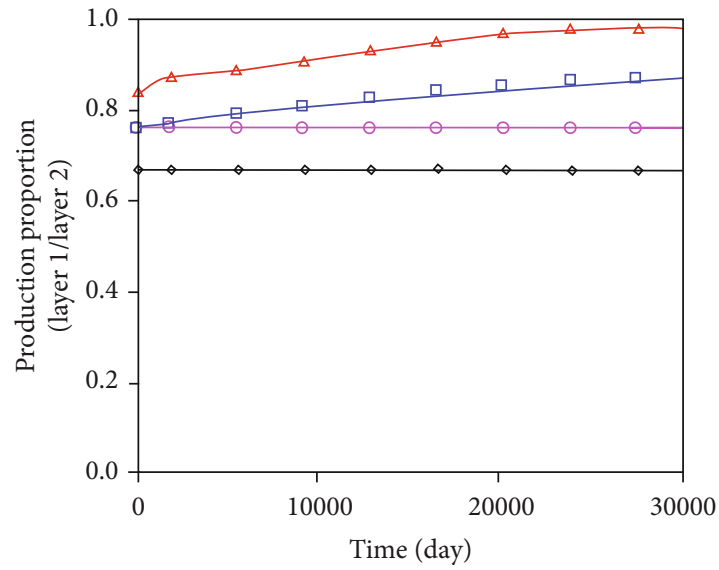

$$
\begin{aligned}
& \triangle \text { Numerical simulation results curve } \\
& \rightarrow \text { DPSM results curve } \\
& \rightarrow \text { KH results curve } \\
& \rightarrow \text { Original methods based on } \\
& \text { catastrophe theory }
\end{aligned}
$$

Figure 8: Comparison of results between the DPSM, KH method, and numerical simulation.

3.3. DPSM Validation. In order to prove the accuracy of DPSM, the results of DPSM, KH method, and numerical simulation are compared. The detailed calculation process and the data are given above, and the calculation is ran by a computer. Figure 8 displays the comparison of results between the DPSM, KH method, and numerical simulation.

In Figure 8, at the beginning of the production stage, the ratio of numerical simulation has a distinct variation. With the increase of oil production time, the curve of the numerical simulation raises rapidly. The calculation result of $\mathrm{KH}$ is a straight line that does not change with time. At the initial moment, the error is $17 \%$. The error increases quickly with time. The error calculated at the end of production time is $30 \%$. The error calculated by the original method based on catastrophe theory is $8 \%$. As the production process progresses, the error gradually increases up to $22 \%$. The results of DPSM are closer to those of numerical simulation and have the same raise trend in the process of production. The error of calculation remains around $8 \%$.

The KH method only calculates the splitting coefficient based on layer permeability and effective thickness, which generates a rough result. Moreover, because the selected parameters are static parameters, they cannot be adapted to the change of the practical yield splitting coefficient in the production process. The error increases as the production process progresses. The original method based on catastrophe theory has a higher accuracy because of having more parameters for comprehensiveness. Similarly, because the selected parameters are static, the accuracy in the initial stage of production is higher and it is opposite in the later stage of production.

The new method proposed in this paper draws on the characteristics of the original splitting method based on the catastrophe theory and uses the MBE to calculate the dynamic parameters of the production process. This method achieves the purpose of calculating the splitting coefficient 
dynamically. Compared with the $\mathrm{KH}$ method and the old splitting method based on the catastrophe theory, the method in this paper inherits the advantages of the other two methods. It increases the accuracy of instantaneous time by taking into account many factors. Through the application of dynamic parameters, the precision in the production process is increased. It is simple and intuitional for the evaluation of production splitting. This method provides a new idea for production splitting, and it will directly affect the accuracy of reservoir performance analysis and fine reservoir description.

\section{Conclusion}

(1) For a multilayer coproduction well in a tight gas reservoir, the production ratio of layers is controlled by many factors. The effect of different factors on production is shown in the direction and degree of action. The splitting method that only considers less factors, such as the KH method, is not accurate and cannot meet the practical production needs. In the process of production splitting, the effect of various factors on the layer production ratio should be considered as much as possible

(2) During the production of the reservoir, reservoir parameters will change as it shows that the production of a small layer changes with time. The production splitting results based only on static data, such as the result of the $\mathrm{KH}$ method, cannot accurately represent the practical production ratio during the production process. It is necessary to combine dynamic factors with static factors to obtain accurate production splitting results

(3) A novel DPSM method to split the production of multilayer coproduction wells is proposed. Compared with the traditional $\mathrm{KH}$ method and static method which is based on the catastrophe theory, the proposed method takes into account both dynamic and static factors. And the calculation result changes dynamically with the production process, which is more in line with the numerical simulation results, and keeps the error within 10\%. This method has a certain practical value and can be used for the coproduction of a tight gas reservoir and can improve the accuracy of reservoir performance analysis and detailed descriptions

(4) In the process of production splitting, the combination of the catastrophe theory, material balance equation (MBE), and iterative calculation method can achieve dynamic results while synthesizing multiple factors. It is proved that the catastrophe theory has a great prospect of production splitting. This prepares the ground for production splitting of more factors through catastrophe theory. It shows the feasibility of production splitting by integrating multiple theories and provides a new idea by using multiple theories

\section{Nomenclature}

$G_{w}$ : Cumulative water production, $\mathrm{m}^{3}$

A: $\quad$ Gas-bearing area, $\mathrm{km}^{2}$

$h$ : $\quad$ Average thickness, $m$

$\Phi$ : $\quad$ Average porosity, \%

$S_{g}: \quad$ Average gas saturation, $\%$

$S_{g i}:$ Initial gas saturation, $\%$

$B_{w i}:$ Underwater volume ratio, 1.005

$K_{g}:$ Effective permeability, $\mathrm{mD}$

$K_{r g}:$ Relative permeability, $\mathrm{mD}$

$K: \quad$ Absolute permeability, $\mathrm{mD}$.

\section{Data Availability}

The data used to support the findings of this study are intersected within the article.

\section{Conflicts of Interest}

The authors declare that there is no conflict of interests regarding the publication of this paper.

\section{Acknowledgments}

The authors would like to acknowledge the financial support from the National Natural Science Foundation of China (51774256).

\section{References}

[1] X. Q. Pang, C. Z. Jia, J. Q. Chen et al., "A unified model for the formation and distribution of both conventional and unconventional hydrocarbon reservoirs," Geoscience Frontiers, vol. 12, no. 2, pp. 695-711, 2021.

[2] G. X. Li and R. K. Zhu, "Progress, challenges and key issues of unconventional oil and gas development of CNPC," China Petroleum Exploration, vol. 25, pp. 1-13, 2020.

[3] J. Tian, Y. L. Kang, L. J. You, N. Jia, Z. Xi, and P. Luo, "Investigation on water phase trapping mechanisms in tight gas reservoirs: pore-scale visualization observation and core-scale flooding analysis," Journal of Petroleum Science and Engineering, vol. 198, p. 108185, 2021.

[4] F. Yang, X. H. Dong, A. Amann-Hildenbrand, E. Fathi, and D. Elsworth, "Petrophysics and fluid transport in shales and tight reservoirs," Geofluids, vol. 2018, Article ID 5691953, 3 pages, 2018.

[5] T. Q. Xia, E. Dontsov, Z. W. Chen, F. Zhang, M. Wei, and $\mathrm{X}$. Kong, "Fluid flow in unconventional gas reservoirs," Geofluids, vol. 2018, Article ID 2178582, 2 pages, 2018.

[6] Z. D. Li, H. Pang, Z. Li, H. X. Zhang, D. J. Wang, and J. Li, "Sand production prediction model for tight sandstone oil reservoirs," Geofluids, vol. 2020, Article ID 8832703, 7 pages, 2020.

[7] Y. L. Wei, W. Liu, and Z. K. Hou, "Experimental study on the anisotropic characteristics and engineering application of tight sandstone," Advances in Materials Science and Engineering, vol. 2021, 12 pages, 2021.

[8] S. H. Zhao, Y. B. Wang, Y. Li et al., "Co-production of tight gas and coalbed methane from single wellbore: a simulation study from Northeastern Ordos Basin, China," Natural Resources Research, vol. 30, no. 2, pp. 1597-1612, 2021. 
[9] G. F. Liu, Z. Meng, D. Y. Luo, J. Wang, D. Gu, and D. Yang, "Experimental evaluation of interlayer interference during commingled production in a tight sandstone gas reservoir with multi-pressure systems," Fuel, vol. 262, article 116557, 2020.

[10] J. L. Guo, F. K. Meng, A. L. Jia et al., "Production behavior evaluation on multilayer commingled stress-sensitive carbonate gas reservoir," Energy Exploration \& Exploitation, vol. 39, no. 1, pp. 86-107, 2021.

[11] X. M. Cao, F. Zhang, and Y. T. Cai, "Research on a new production split method of thin interbedded sandstone reservoirs," Science Technology and Engineering, vol. 14, pp. 166171, 2014.

[12] Y. Jiang, Q. Zhou, F. Guan, and K. Zhu, "Splitting method of the commingled gas well production based on gas and water phase permeabilities," Petroleum Geology \& Oilfield Development in Daqing, vol. 34, pp. 73-76, 2015.

[13] J. C. Zhang and X. Y. Wang, "The method of oil production splitting based on water saturation and its application," Journal of Zhejiang University (Science Edition), vol. 42, pp. 626630, 2015.

[14] H. L. Yu, S. Q. Cheng, L. J. Zhang, G. Q. Zhang, and J. L. Wang, "Plate approach to determine separate layer remaining oil saturation according to water cut of produces," Petroleum Geology \& Oilfield Development in Daqing, vol. 30, pp. 105-109, 2011.

[15] Y. L. Li, Z. P. Yang, D. S. Pan, S. J. Yue, and L. X. Zhi, "A production splitting method of sandstone reservoir considering the change of relative permeability," Journal of Xi'an Shiyou University (Natural Science Edition), vol. 35, pp. 72-76, 2020.

[16] Z. P. Yang, S. J. Yue, C. L. Zheng, X. Z. Liu, and G. X. Chen, "Production split method restricted synthetically by multifactors in thin interbed sandstone reservoirs," Lithologic Reservoirs, vol. 30, pp. 117-124, 2018.

[17] H. S. Prabowo and M. A. Rinadi, "Production allocation method for commingled gas completions," in SPE International Meeting on Petroleum Engineering, pp. 261-269, Beijing, PR China, November 1995.

[18] Y. Zhu, J. Z. Xie, W. H. Yang, and L. H. Hou, "Method for improving history matching precision of reservoir numerical simulation," Petroleum Exploration and Development, vol. 35, no. 2, pp. 225-229, 2008.

[19] M. K. V. Lerma, "Analytical method to predict waterflood performance," in SPE Western Regional/AAPG Pacific Section Joint Meeting, pp. 1-12, Long Beach, California, U.S.A., May 2003.

[20] X. D. Wang and C. Q. Liu, "Production analysis on commingled production wells," Oil Drilling \& Production Technology, vol. 21, no. 2, pp. 56-61, 1999.

[21] P. C. Liu, X. D. Wang, X. C. Hou, L. FH, and D. HW, "Productivity analysis of commingled production in circle-sealing reservoir with infinite conductive vertical fractures," Xinjiang Petroleum Geology, vol. 25, pp. 297-300, 2004.

[22] Q. G. Liu, H. Wang, R. C. Wang, and X. C. Li, "A computing method for layered production contribution and affecting factors analyzing in commingled gas reservoirs," Journal of Southwest Petroleum University (Science \& Technology Edition), vol. 32, pp. 80-84, 2010.

[23] M. A. Sallam and A. H. El-Banbi, "Analysis of multi-layered commingled and compartmentalized gas reservoirs," Journal of Petroleum Exploration and Production Technology, vol. 8, no. 4, pp. 1573-1586, 2018.
[24] S. Zavaleta, P. M. Adrian, and M. Michel, "Estimation of OGIP in a water-drive gas reservoir coupling dynamic material balance and Fetkovich aquifer model," in SPE Trinidad and Tobago Section Energy Resources Conference, p. 11, Port of Spain, Trinidad and Tobago, June 2018.

[25] Q. H. Hu, X. Wang, Y. M. Tan, H. Yang, F. Zhang, and M. Lu, "The production split method in multilayer reservoir based on grey relational analysis," in IOP Conference Series: Earth and Environmental Science, Volume 113, 3rd International Conference on Advances in Energy Resources and Environment Engineering, p. 8, Harbin, China, 2018.

[26] R. R. A. Rasoul, A. Daoud, and E. S. A. El Tayeb, "Production allocation in multi-layers gas producing wells using temperature measurements with the application of a genetic algorithm," Petroleum Science and Technology, vol. 32, no. 3, pp. 363-370, 2014.

[27] D. H. Gu, G. F. Cui, G. F. Liu, and D. D. Quan, "A new method of production splitting for multilayer commingled gas well," Nature Gas Geoscience, vol. 27, pp. 1346-1351, 2016.

[28] L. Tian, Z. Q. Shen, L. Liu, and M. Wang, "Production split method for multilayer-superimposed tight sandstone gas reservoirs," Natural Gas Exploration \& Development, vol. 39, pp. 41-44, 2016.

[29] L. D. Mi, X. Y. Hu, Y. Jia, and Q. Liu, "A novel dynamic production splitting method based on the catastrophe theory," in SPE Middle East Oil and Gas Show and Conference, p. 8, Manama, Bahrain, March 2019.

[30] R. Y. Cao, L. Y. Ye, Q. H. Lei, X. Chen, Y. Z. Ma, and X. Huang, "Gas-water flow behavior in water-bearing tight gas reservoirs," Geofluids, vol. 2017, Article ID 9745795, 16 pages, 2017.

[31] Y. H. Shen, M. M. Meng, T. Liu, H. Ge, and Y. Zhang, "Impact of petrophysical properties on hydraulic fracturing and development in tight volcanic gas reservoirs," Geofluids, vol. 2017, Article ID 5235140, 13 pages, 2017.

[32] J. Zhang, F. F. Fang, W. J. Shen, H. Liu, S. Gao, and L. Ye, "Experimental study on the effective utilization of reserves in tight sandstone gas reservoirs and their applications," Geofluids, vol. 2020, Article ID 8854299, 13 pages, 2020.

[33] Y. D. Cai, Z. J. Pan, D. M. Liu et al., "Effects of pressure and temperature on gas diffusion and flow for primary and enhanced coalbed methane recovery," Energy Exploration \& Exploitation, vol. 32, no. 4, pp. 601-619, 2014.

[34] L. Wang, C. S. Huang, X. G. Yang, Z. Chai, and B. Shi, "Effects of temperature-dependent properties on natural convection of power-law nanofluids in rectangular cavities with sinusoidal temperature distribution," International Journal of Heat and Mass Transfer, vol. 128, pp. 688-699, 2019.

[35] J. S. Wu, Y. P. Bai, H. H. Zhao, X. Hu, and V. Cozzani, “A quantitative LNG risk assessment model based on integrated Bayesian- Catastrophe-EPE method," Safety Science, vol. 137, article 105184, 2021.

[36] M. Haragus and G. Iooss, "Bifurcation theory," in Encyclopedia of Mathematical Physics, Elsevier Ltd, 2006.

[37] L. Mattar and D. Anderson, "Dynamic material balance (oil or gas-in-place without shut-ins)," in Petroleum Society's 6th Canadian International Petroleum Conference (56th Annual Technical Meeting), pp. 7-9, Calgary, Alberta, Canada, June 2005.

[38] X. F. Tian, L. S. Cheng, Y. Q. Yan, H. Liu, W. Zhao, and Q. Guo, "An improved solution to estimate relative permeability in tight oil reservoirs," Journal of Petroleum Exploration and Production Technology, vol. 5, no. 3, pp. 305-314, 2015. 